\title{
Unidirectional self-driving liquid droplet transport on a monolayer graphene-covered textured substrate
}

\author{
Zhongqiang Zhang ${ }^{1,2,3,4}$, Xinfeng Guo ${ }^{1}$, Huayuan Tang ${ }^{3}$, Jianning Ding ${ }^{1,4}$, Yong-Gang \\ Zheng ${ }^{3 *}$, and Shaofan $\mathrm{Li}^{2}$ *
}

${ }^{1}$ Micro/Nano Science and Technology Center, Jiangsu University, Zhenjiang, 212013, P.R. China

${ }^{2}$ Department of Civil and Environmental Engineering, University of California, Berkeley, CA 94720-1710, USA

${ }^{3}$ State Key Laboratory of Structural Analysis for Industrial Equipment, Department of Engineering Mechanics, Faculty of Vehicle Engineering and Mechanics, Dalian University of Technology, Dalian 116024, P. R. China

${ }^{4}$ Jiangsu Collaborative Innovation Center of Photovoltaic Science and Engineering, Changzhou University, Changzhou, 213164, P.R. China

\footnotetext{
* Corresponding author. Tel.: +86-411-84708751

E-mail address: zhengyg@ dlut.edu.cn (Yong-Gang Zheng)

* Corresponding author. Tel.: (510) 642-5362

E-mail address: shaofan@berkeley.edu (Shaofan Li)
} 


\section{Section 1. Comparison of Self-Driving Behaviors of $\mathrm{Hg}$ Droplets on Both Graphene-Cu}

\section{and Pure Cu grooves}

Based the MD model in Figure 2 (main text), we further constructed a long-groove model (see Figure S1) with a longer groove in $\mathrm{x}$ direction without changing the width gradient of $7^{\circ}$, and displaced the $\mathrm{Hg}$ droplet to a narrower end to observe its self-driving behaviors using MD simulations. Figure S2 plots the driving velocity and displacement versus time to compare the self-driving results between the pure $\mathrm{Cu}$ and graphene- $\mathrm{Cu}$ models. It shows obviously that the existence of monolayer graphene significantly improves the self-driving efficiency of $\mathrm{Hg}$ droplet. Moreover, the $\mathrm{Hg}$ droplet break away from one side wall of the groove due to the larger velocity caused by a longer acceleration stage, which is consistent with the result in Figure S2a.

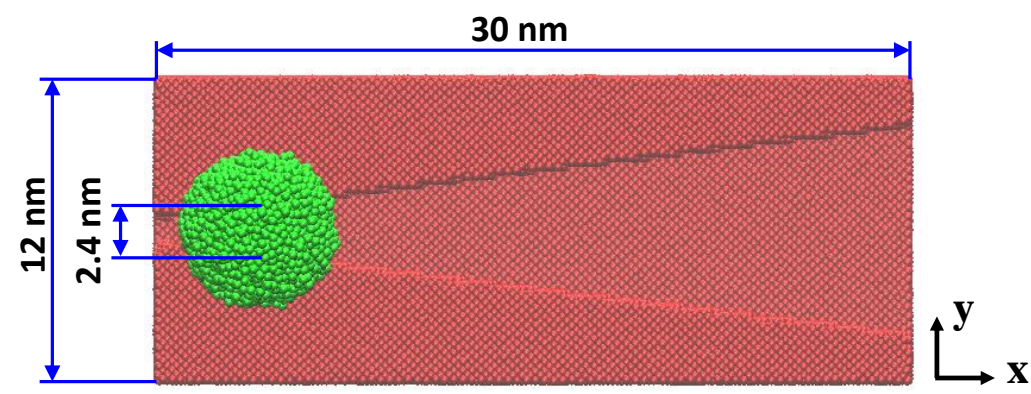

Figure S1. Top view of a $\mathrm{Hg}$ droplet with a diameter of $d=5.8 \mathrm{~nm}$ on a pure $\mathrm{Cu}$ gradient groove with length of $30 \mathrm{~nm}$ in $\mathrm{x}$ direction. The width of groove corresponding to the initial position of $\mathrm{Hg}$ droplet is $2.4 \mathrm{~nm}$.

(a)

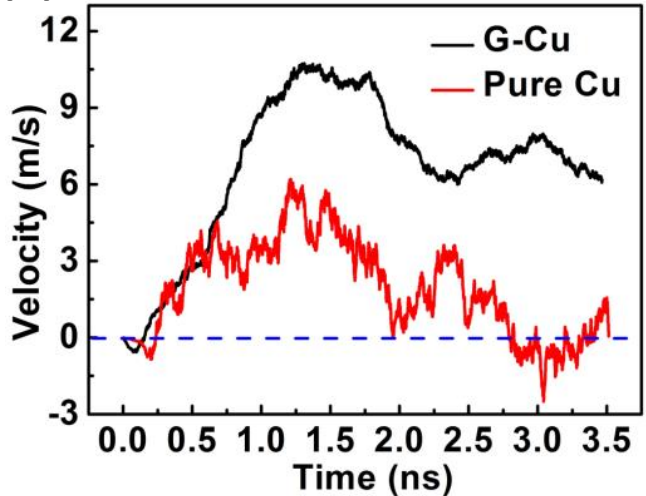

(b)

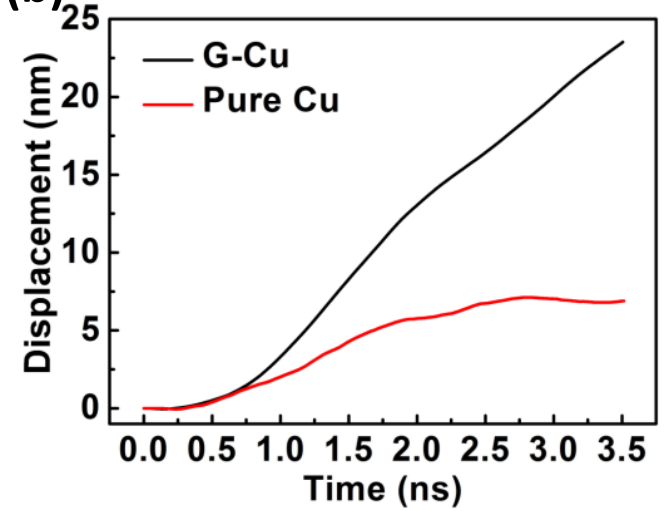

Figure S2. Comparisons of (a) moving velocity and (b) displacement versus time for a $5.8 \mathrm{~nm}$-diameter $\mathrm{Hg}$ droplet on both the pure $\mathrm{Cu}$ and Graphene-Cu groove models. The structural parameters of groove can be found in Figure R6. 


\section{Section 2. Computational Method of Contact Angle}

MD model is an $\mathrm{Hg}$ droplet on the graphene-covered smooth $\mathrm{Cu}$ surface. In the case of $d$ $=5.8 \mathrm{~nm}$, as shown in Figure $\mathrm{S} 3$, the radius of the $\mathrm{Hg}$ droplet $r$ can be obtained by fitting the coordinates of the $\mathrm{Hg}$ atoms at the boundary of droplet. Hence, the contact angle $\alpha$ can be calculated through the geometrical relationship between $r$ and contact radius $r_{\mathrm{i}}$. In this work, the contact angle $\alpha=155.6^{\circ}$ is an 5 times average value for $d=5.8,6.6,7.2,8.2$, and $8.8 \mathrm{~nm}$ (Table S1).

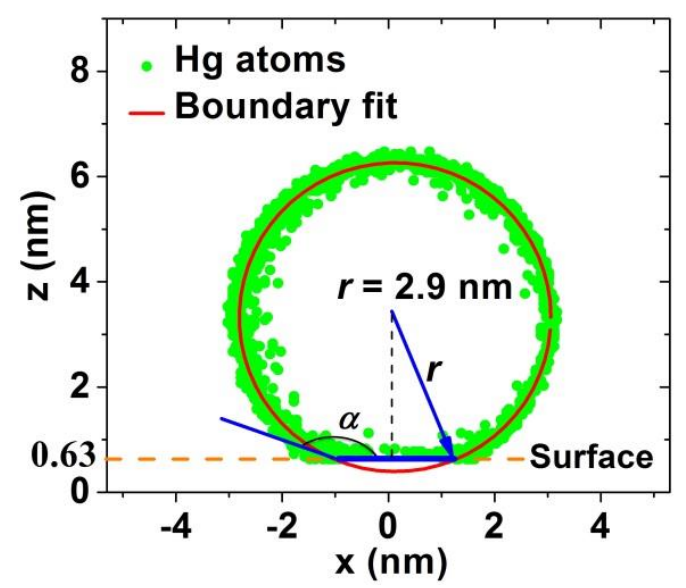

Figure S3. Schematic of a $\mathrm{Hg}$ droplet on the smooth graphene-Cu substrate in the case of $d=5.8 \mathrm{~nm}$. Green points are the $\mathrm{Hg}$ atoms at the boundary of droplet, the red circle is the boundary fit of $\mathrm{Hg}$ droplet, and the orange dashed line is the surface of the substrate. The blue arrow is the radius $r=2.9 \mathrm{~nm}$, and the blue solid line is the contact line of the droplet on the substrate. $\alpha$ is the contact angle.

Table S1. Data of the diameter of droplets $d$, the contact radius $r_{\mathrm{i}}$, and the contact angle $\alpha$ for different size of $\mathrm{Hg}$ droplets.

\begin{tabular}{llllll}
\hline$d(\mathrm{~nm})$ & 5.8 & 6.6 & 7.2 & 8.2 & 8.8 \\
$r_{\mathrm{i}}(\mathrm{nm})$ & 1.158 & 1.277 & 1.493 & 1.732 & 1.907 \\
$\alpha\left({ }^{\circ}\right)$ & 156.45 & 157.22 & 155.48 & 155.00 & 153.98 \\
\hline
\end{tabular}




\section{Section 3. Calculation of Pressure Difference within Hg Droplets}

The surfaces of the adsorbed portion of the liquid $\mathrm{Hg}$ droplets confined by two lateral groove walls are assumed to be cylindrical approximately with radii of curvature $R_{1}$ and $R_{2}$. Then, the curvatures of its trailing and advancing menisci can be obtained in terms of the geometric relationships (Figure S4).

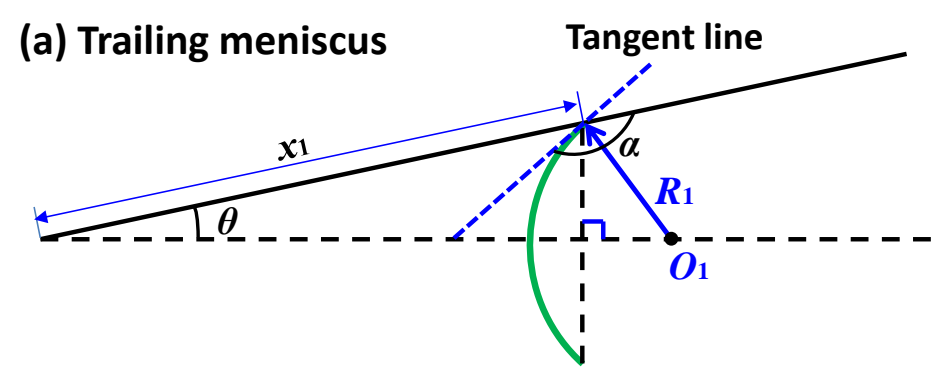

\section{(b) Advancing meniscus}

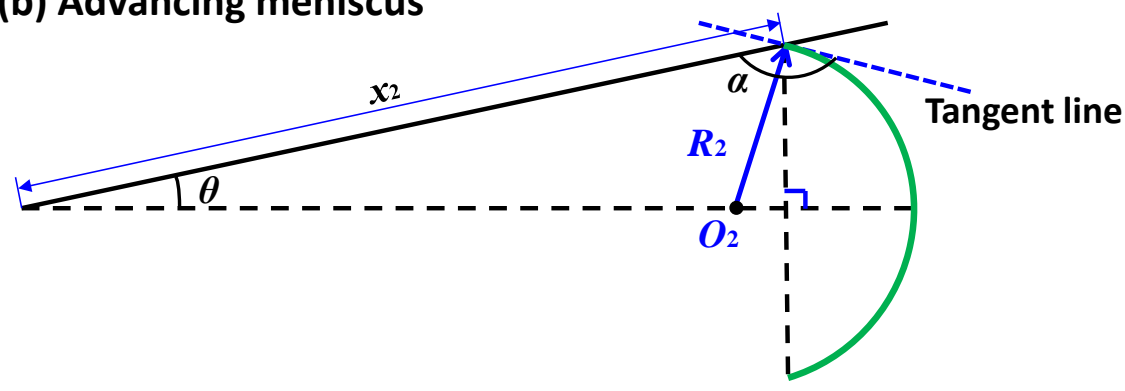

Figure S4 Schematics of the geometric relationships for the droplet curvatures of trailing and advancing menisci.

From Figure S4a, we find that $\cos [\pi-(\alpha-\theta)]=\frac{x_{1} \sin \theta}{R_{1}}$. So the curvature of the trailing meniscus is $R_{1}^{-1}=-\cos (\alpha-\theta) /\left(x_{1} \sin \theta\right)$. Similarly, the geometric relationship of $\cos [\pi-(\alpha+\theta)]=\frac{x_{2} \sin \theta}{R_{2}}$ can be obtained, and then the curvature of the advancing meniscus can be calculated by $R_{2}^{-1}=-\cos (\alpha+\theta) /\left(x_{2} \sin \theta\right)$ (Figure $\mathrm{S} 4 \mathrm{~b}$ ).

Subsequently, in terms of Young-Laplace equation, the pressure inside the droplet corresponding to the trailing meniscus $P_{1}$ can be obtained by [1]

$$
P_{1}=-\gamma \frac{\cos (\alpha-\theta)}{x_{1} \sin \theta}
$$

Also, the pressure inside the droplet corresponding to the advancing meniscus $P_{2}$ can be 
written by

$$
P_{2}=-\gamma \frac{\cos (\alpha+\theta)}{x_{2} \sin \theta}
$$

Consequently, the pressure different $\Delta P$ within the $\mathrm{Hg}$ droplets in $+x$ direction can be written as 1,2

$$
\Delta P=P_{1}-P_{2}=\left[\frac{\cos (\alpha+\theta)}{x_{2} \sin \theta}-\frac{\cos (\alpha-\theta)}{x_{1} \sin \theta}\right]
$$




\section{Section 4. Details of Continuum Model}

In the continuum model, a droplet, which is modeled as a thin shell with surface tension $\gamma$, is adhered on the substrate with a groove of constant width $w$. The interactions between the atoms of the droplet and the substrate are described by the Lennard-Jones (LJ) potential $U_{\mathrm{LJ}}(r)=4 \varepsilon\left[\left(\frac{\sigma}{r}\right)^{12}-\left(\frac{\sigma}{r}\right)^{6}\right]$. Since the interfacial energy is mainly determined by the interfaces properties of material, we can simplify the calculation of adhesion energy in the continuum model by integrating the LJ potentials over the surface atoms.

The interfacial energy $\mathrm{d} e_{\mathrm{Hg}-\mathrm{S}}$ between a surface element $\mathrm{d} A_{\mathrm{Hg}}$ on the droplet and a surface element $\mathrm{d} x \mathrm{~d} y$ on the substrate is given by ${ }^{3}$

$$
\mathrm{d} e_{\mathrm{Hg}-\mathrm{S}}=4 \varepsilon \rho_{\mathrm{Hg}} \rho_{\mathrm{S}} \mathrm{d} A_{\mathrm{Hg}} \mathrm{d} x \mathrm{~d} y\left[\left(\frac{\sigma}{r}\right)^{12}-\left(\frac{\sigma}{r}\right)^{6}\right]
$$

where $\rho_{\mathrm{Hg}}$ and $\rho_{\mathrm{S}}$ are the atom densities of the droplet and the substrate surface, respectively. $r$ is the distance between $\mathrm{d} A_{\mathrm{Hg}}$ on the droplet and $\mathrm{d} x \mathrm{~d} y$ on the substrate. Then, the total interfacial energy between $\mathrm{d} A_{\mathrm{Hg}}$ and the whole substrate $e_{\mathrm{Hg}-\mathrm{S}}$ is given by

$$
e_{\mathrm{Hg}-\mathrm{S}}=4 \varepsilon \rho_{\mathrm{Hg}} \rho_{\mathrm{S}} \int_{-\infty}^{\infty} \int_{-\infty}^{\infty}\left[\left(\frac{\sigma}{r}\right)^{12}-\left(\frac{\sigma}{r}\right)^{6}\right] \mathrm{d} x \mathrm{~d} y \mathrm{~d} A_{\mathrm{Hg}}
$$

Since it is assumed that the width of the groove is constant in the continuum model, eq S5 can be integrated with respect to $y$ analytically, which gives ${ }^{4}$

$$
e_{\mathrm{Hg}-\mathrm{S}}=\pi \varepsilon \rho_{\mathrm{Hg}} \rho_{\mathrm{S}} \sigma \int_{-\infty}^{\infty}\left[\frac{63}{64}\left(\frac{\sigma}{l}\right)^{11}-\frac{3}{2}\left(\frac{\sigma}{l}\right)^{5}\right] \mathrm{d} x \mathrm{~d} A_{\mathrm{Hg}}
$$

where $l$ is the distance between $\mathrm{d} A_{\mathrm{Hg}}$ and an infinitely long line $\mathrm{d} x$. Note that $\varepsilon, \rho_{\mathrm{Hg}}$ and $\rho_{\mathrm{S}}$ in eq (S6) remain to be determined. To avoid the difficulties, we further consider the interfacial energy between $\mathrm{d} A_{\mathrm{Hg}}$ and an infinitely large flat substrate. Integrating eq (S6) with respect to $x$ gives 


$$
e_{\mathrm{Hg}-\mathrm{S}}=4 \pi \varepsilon \rho_{\mathrm{Hg}} \rho_{\mathrm{S}}\left[\frac{\sigma^{12}}{5 h^{10}}-\frac{\sigma^{6}}{2 h^{4}}\right] \mathrm{d} A_{\mathrm{Hg}}
$$

where $h$ is the distance between $\mathrm{d} A_{\mathrm{Hg}}$ and the flat substrate. By minimizing the interfacial energy with respect $h$, i.e., $\mathrm{d} e_{\mathrm{Hg}-\mathrm{S}} / \mathrm{d} h=0$, the equilibrium distance $h_{\text {eq }}$ between $\mathrm{d} A_{\mathrm{Hg}}$ and the flat substrate is found to be

$$
h_{\mathrm{eq}}=\sigma
$$

Then, the interfacial energy at the equilibrium distance is

$$
e_{\mathrm{eq}}=-u \mathrm{~d} A_{\mathrm{Hg}}
$$

where $u=\frac{6}{5} \pi \varepsilon \rho_{\mathrm{Hg}} \rho_{\mathrm{S}} h_{\mathrm{eq}}^{2}$ is the interfacial energy per unit area at the equilibrium distance, which is referred as the interfacial strength. Combining eqs (S6), (S8) and (S9), the interfacial energy between $\mathrm{d} A_{\mathrm{Hg}}$ and an infinitely long line $\mathrm{d} x$ can be expressed as

$$
e_{\mathrm{Hg}-\mathrm{S}}=\frac{5 u}{128 h_{\mathrm{eq}}} \int_{-\infty}^{\infty}\left[21\left(\frac{h_{\mathrm{eq}}}{l}\right)^{11}-32\left(\frac{h_{\mathrm{eq}}}{l}\right)^{5}\right] \mathrm{d} x \mathrm{~d} A_{\mathrm{Hg}} .
$$

Thus, the total interfacial energy between the droplet and the substrate is

$$
E_{\mathrm{Hg}-\mathrm{S}}=\frac{5 u}{128 h_{\mathrm{eq}}} \int_{A_{\mathrm{Hg}}} \int_{-\infty}^{\infty}\left[21\left(\frac{h_{\mathrm{eq}}}{l}\right)^{11}-32\left(\frac{h_{\mathrm{eq}}}{l}\right)^{5}\right] \mathrm{d} x \mathrm{~d} A_{\mathrm{Hg}} .
$$

The parameters $u$ and $h_{\mathrm{eq}}$ in eq (S11) are extracted from the molecular dynamics simulations. Specifically, a droplet is equilibrated on a flat substrate without groove and the equilibrium distance $h_{\mathrm{eq}}$, the total interfacial energy $E_{\mathrm{eq}}$ and the interfacial area $A_{\mathrm{eq}}$ are measured. Then, the interfacial strength can be obtained by $u=E_{\mathrm{eq}} / A_{\mathrm{eq}}$. In this continuum model, we chose $h_{\mathrm{eq}}=0.308 \mathrm{~nm}$ and $u(d)$ are obtained from MD results in Section 2 of Supporting Information. 


\section{Section 5. Calculations of Equilibrium Distance and Interfacial Strength}

In this work, the equilibrium distance between liquid $\mathrm{Hg}$ and the smooth graphene-Cu substrate is the normal distance between the graphene surface to the first leap of the number density of liquid $\mathrm{Hg}$ at the liquid-solid interface. Then the equilibrium distance $h_{\mathrm{eq}}=$ $0.308 \mathrm{~nm}$. The magnitude of interaction energy $E$ between $\mathrm{Hg}$ droplets and the smooth graphene-Cu substrate can be directly obtained by the command 'compute group/group' of the software LAMMPS. The MD data of $E$ and the contact area $A$ for different size of $\mathrm{Hg}$ droplets on the substrate are listed in Table S2. Then, the interfacial strength u can be calculated by the formula $u=E / A$. It can be seen that the interfacial strength decrease with the increase in the diameter of droplets, as shown in Figure S5.

Table S2. The interfacial energy $E$, the contact area $A$, and the interfacial strength $U$ as functions of the diameter of droplets $d$.

\begin{tabular}{llllll}
\hline$d(\mathrm{~nm})$ & 5.8 & 6.6 & 7.2 & 8.2 & 8.8 \\
$E(\mathrm{eV})$ & 2.276 & 2.577 & 3.140 & 3.918 & 4.660 \\
$A\left(\mathrm{~nm}^{2}\right)$ & 4.21 & 5.12 & 7.00 & 9.42 & 11.42 \\
$u\left(\mathrm{eV} / \mathrm{nm}^{2}\right)$ & 0.5406 & 0.5033 & 0.4486 & 0.4159 & 0.4081 \\
\hline
\end{tabular}

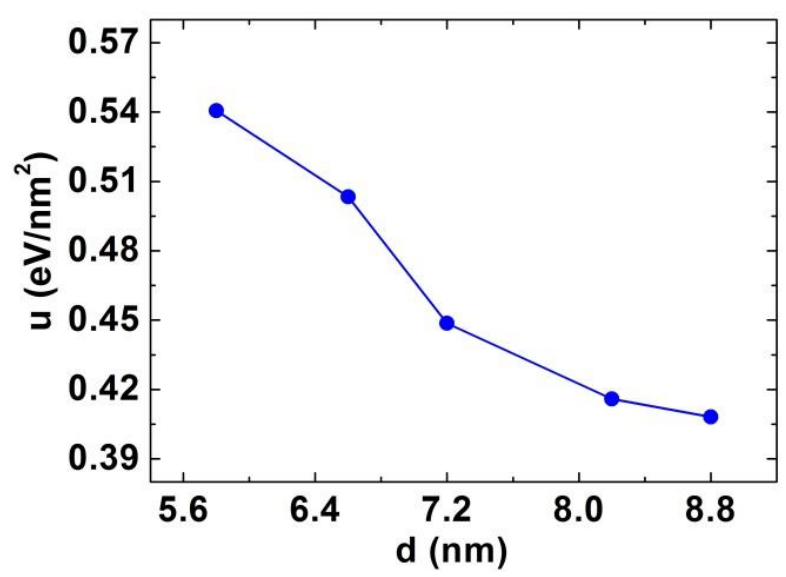

Figure S5. Interfacial strength $u$ between $\mathrm{Hg}$ droplet and graphene-Cu substrate as a function of the diameter $d$ of droplets. 


\section{References}

(1) Adamson, A. W. Physical Chemistry of Surfaces, 5th ed.; John Wiley \& Sons: New York, 1990.

(2) Luo, C.; Heng, X.; Xiang, M. Behavior of a Liquid Drop between Two Nonparallel Plates. Langmuir 2014, 30, 8373-8380.

(3) Jiang, L. Y.; Huang, Y.; Jiang, H.; Ravichandran, G.; Gao, H.; Hwang, K. C.; Liu, B. A Cohesive Law for Carbon Nanotube/Polymer Interfaces Based on the Van der Waals Force. Journal of the Mechanics and Physics of Solids 2006, 54, 2436-2452

(4) Zheng, Y. G.; Tang, H. Y.; Ye, H. F.; Zhang, H. W. Adhesion and Bending Rigidity-Mediated Wrapping of Carbon Nanotubes by A Substrate-Supported Cell Membrane. RSC Adv. 2015, 5, 43772-43779 